\title{
Complex Ginzburg-Landau equation in the presence of walls and corners
}

\author{
Víctor M. Eguíluz, ${ }^{1,2, *}$ Emilio Hernández-García, ${ }^{2}$ and Oreste Piro ${ }^{2}$ \\ ${ }^{1}$ Center for Chaos and Turbulence Studies, The Niels Bohr Institute, Blegdamsvej 17, DK2100 Copenhagen D, Denmark \\ ${ }^{2}$ Instituto Mediterráneo de Estudios Avanzados IMEDEA (CSIC-UIB), E-07071 Palma de Mallorca, Spain
}

(Received 8 December 2000; revised manuscript received 10 May 2001; published 14 August 2001)

\begin{abstract}
We investigate the influence of walls and corners (with Dirichlet and Neumann boundary conditions) in the evolution of two-dimensional autooscillating fields described by the complex Ginzburg-Landau equation. Analytical solutions are found, and arguments provided, to show that Dirichlet walls introduce strong selection mechanisms for the wave pattern. Corners between walls provide additional synchronization mechanisms and associated selection criteria. The numerical results fit well with the theoretical predictions in the parameter range studied.
\end{abstract}

DOI: 10.1103/PhysRevE.64.036205

PACS number(s): 05.45.-a, 47.54.+r

\section{INTRODUCTION}

Spatially extended nonlinear dynamical systems display an amazing variety of behavior, including pattern formation, self-organization, and spatiotemporal chaos [1-4]. Much effort has been devoted to the characterization of the different dynamical regimes and the transitions between them for model equations such as the complex Ginzburg-Landau equation (CGLE) [5]. This is an equation for a complex field $A(\mathbf{x}, t)$ that conveniently rescaled reads

$$
\partial_{t} A=A+(1+i \alpha) \nabla^{2} A-(1+i \beta)|A|^{2} A,
$$

$\alpha$ and $\beta$ are real parameters. This equation describes the onset of an oscillatory regime through the Hopf bifurcation of a homogeneous state, and it is used generally as a model equation due to the rich variety of its solutions. Binary fluid convection [7], transversally extended lasers [8,9], chemical turbulence [10,11], bluff body wakes [12], the motion of bars in the bed of rivers [13], the jet stream [14], and other systems have been described using the CGLE, or coupled pairs of similar equations. In this paper, we will restrict ourselves to situations well described by a single scalar CGLE in a proper parameter range.

The CGLE admits simple plane-wave solutions. However, for most of the $(\alpha, \beta)$ parameter range, a typical evolution starting from random initial conditions leads to complex, steady or evolving, states. An important ingredient in the description of these dynamical regimes in two-dimensional domains is the interaction of singular points called defects. For our purposes, a defect is just a zero of the complex field $A$, where there is a singularity in the phase $\varphi$ defined by the relation $A=|A| \exp (i \varphi)$. There is a topological charge associated to each defect, $n$, defined by

$$
n=\frac{1}{2 \pi} \oint_{\Gamma} \nabla \varphi \cdot d \mathbf{r}
$$

\footnotetext{
*Email address: victor@imedea.uib.es
}

where $\Gamma$ is a closed path around the defect. The topological nature of the phase singularities implies that $n$ is a positive or negative integer, and that the total topological charge in the two-dimensional system is constant, except for the defects flowing in and out through the boundaries. In the interior of the system, defects can only be created or annihilated in pairs of opposite charge. Spiral defects, i.e., defects around which the lines of constant phase have a spiral form, are typically formed in the CGLE (for $\alpha \neq \beta$ ). The interaction between these spiral structures has attracted much attention [6]. Spiral solutions of a different nature appear, e.g., in excitable media such as the Belousov-Zabotinsky reaction $[15,16]$ and electro-hydrodynamic convection (see, e.g., Ref. [17]).

One important source of defects in real systems are the boundaries. Under some circumstances, walls can introduce defects into the system increasing the amount of disorder in the dynamics. In other situations, the boundaries play the opposite role: they annihilate defects driving the system to a more ordered state. In general, the interplay between these two behaviors and the proper dynamics of the bulk can push the system to configurations different from the ones found under boundary-free conditions (periodic boundary conditions, for instance). However, few studies have addressed the influence of the boundary shapes and boundary conditions on complex dynamics. The importance of these effects in the transverse patterns of laser emission, where aspect ratios are not large, is visible in recent works such as $[18,19]$. In addition, average patterns in Faraday waves and other spatiotemporally chaotic systems have been observed to be sensible to boundary shape $[20,21]$ and boundaries are able even to induce spatial chaos in otherwise nonchaotic systems [22]. All those strong influences of boundaries on the dynamics of extended nonlinear systems [23] provides us with the motivation for a more systematic study of boundary effects on the CGLE performed in this paper.

In Ref. [24], we performed a first numerical exploration of these effects, via computer simulations of the CGLE in circular and rectangular geometries with null-Dirichlet boundary conditions. The results reveal a fundamental role of boundaries in selection mechanisms. In particular, wave emission from Dirichlet walls (i.e., walls where $A=0$ ), and the dominance of corners as pacemakers for the whole sys- 
tem were important observed effects. Understanding the origin of such effects is the main goal of this paper. To achieve it, we will focus first on the effect of a single lateral wall, where the complex field is set to zero, in the selection of the pattern. After this, we will study how the presence of corners (i.e., the intersection of two walls) restricts the family of solutions found previously. It should be noted that we use Dirichlet boundary conditions (and in some parts of this paper, also Neumann boundary conditions) as simple phenomenological conditions to explore deviations with respect to the more commonly used periodic boundaries. A different issue is to establish what are the pertinent boundary conditions arising for the CGLE when it is derived as an amplitude equation in particular physical contexts (for example in optics, fluids, etc.). Some results in this last subject can be found in Refs. [25,26].

In the next section we review previous numerical results on the CGLE in several geometries and boundary conditions. In Sec. III, we summarize analytical solutions in unbounded domains. In Sec. IV, we present analytical and numerical results for the CGLE in the presence of a lateral Dirichlet wall. In Sec. V, we extend our study to the case of corners and in Sec. VI, we finish with our Conclusions.

\section{NUMERICAL OBSERVATIONS}

It is quite evident, and confirmed by our previous study [24], that the effect of boundaries is more noticeable in the parameter regimes for which large correlation lengths are present in the system. In strongly chaotic states with short correlation lengths, the main effects of walls are restricted to boundary layers close to them. Consequently, we restrict here the presentation of our numerical results to the region of parameters for which coherent oscillations extend over nearly the whole system. This happens in most of the Benjamin-Feir stable region in parameter space, that is, for $1+\alpha \beta>0$, but also in other regions close to it. Defects and shocks however disrupt the otherwise ordered plane waves, and its location and structure are strongly dependent on boundaries. In Fig. 1, the CGLE is solved in a square with null-Dirichlet boundary conditions $(A=0)$ [27]. The zeroamplitude boundaries facilitates the formation of defects near the walls. Starting from random initial conditions, defects are actively created in the early stages of the evolution (See Fig. 1). After some time, however, all the points on the boundaries synchronize and oscillate in phase so that plane waves are emitted. Defect formation ceases, and the waves emitted by the walls push the remaining defects towards the central region of the domain. There the defects annihilate in pairs of opposite charge, and as a result of this process, a bound state is formed by the surviving set of equal-charge defects. The orientation of the waves emitted by the boundaries also changes during the evolution. The synchronized emission of the early stages produces wave propagation nearly perpendicular to the boundary, but in the late states the wave vector tilts to some emission angle of approximately $45^{\circ}$. The precise value of this angle depends on both the parameter values and the geometry of the boundaries. The fact that this angle is not exactly $45^{\circ}$ is made evident by the slight mismatch (a)

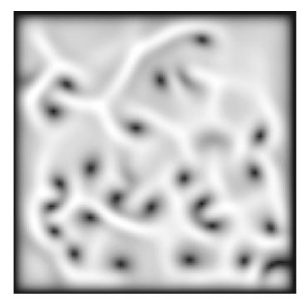

(c)

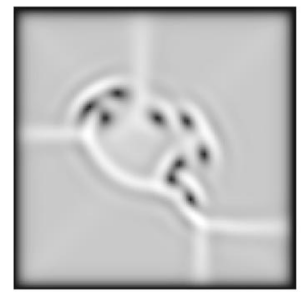

(e)

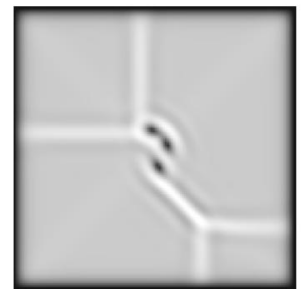

(b)

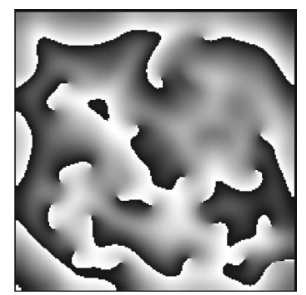

(d)

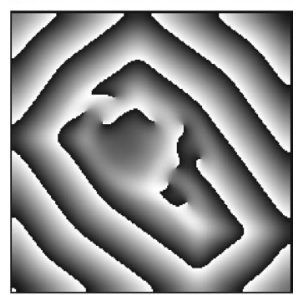

(f)

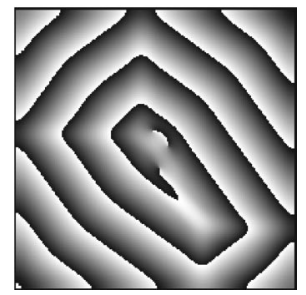

FIG. 1. Time evolution of the solution of the Eq. (1) at arbitrary times with parameter values $\alpha=2, \beta=-0.2$. The initial conditions is random. When the boundary starts emitting waves, the spiral defects are pushed to the interior of the domain and annihilate in pairs of opposite charge. The modulus of the field is plotted in the left column and the phase in the right. Gray scale runs from black (minimum) to white (maximum). The final state (not shown) contains a single defect, as the one in Fig. 2(a).

between the waves coming from orthogonal walls. Finally, the system reaches a frozen state of the type displayed in Fig. 2. The term frozen is used here to denote that the modulus is a steady solution, although the phase is time periodic. More concretely, our frozen configurations are well described by $A(\mathbf{x}, t)=f(\mathbf{x}) e^{-i \omega t}$, with $\omega$ real and $f$ a possibly complex function of position. (a)

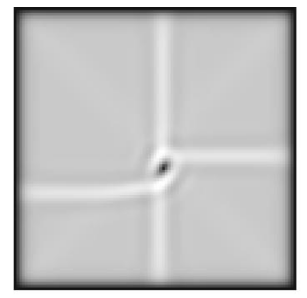

(c)

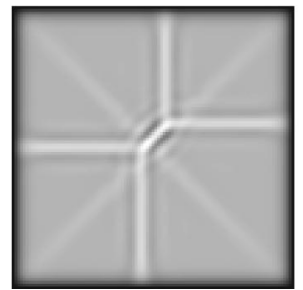

(b)

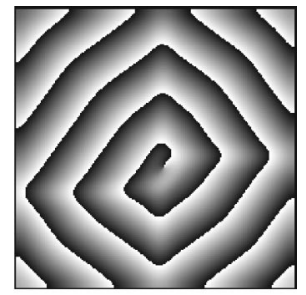

(d)

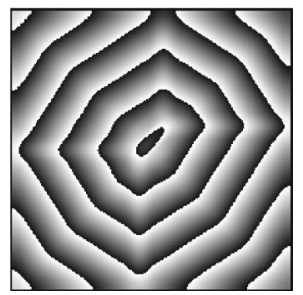

FIG. 2. Frozen structures under null Dirichlet boundary conditions in a square of size $100 \times 100$. Parameter values are $\alpha=2, \beta$ $=-0.2(\mathrm{a}, \mathrm{b})$, and $\alpha=2, \beta=-0.6(\mathrm{c}, \mathrm{d})$. Snapshots of the modulus $|A|$ of the field are shown in the left column and snapshots of the phase in the right column. Gray scale as in Fig. 1. 
(a)

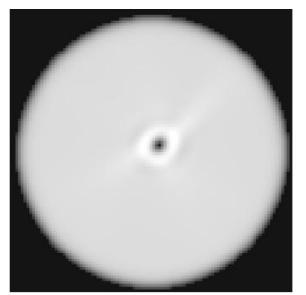

(c)

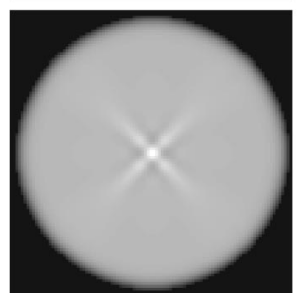

(b)

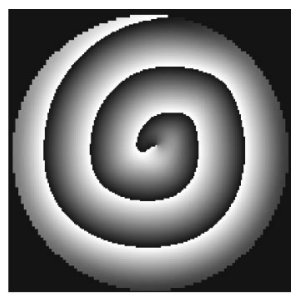

(d)

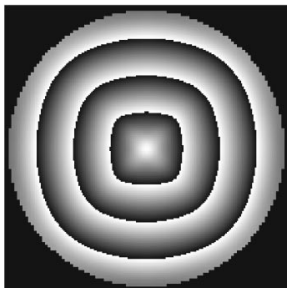

FIG. 3. Frozen structures under null Dirichlet boundary conditions in a circle of diameter 100 for parameter values $\alpha=2, \beta=$ -0.2 (a,b), and $\alpha=2, \beta=-0.6$ (c,d). Snapshots of the modulus $|A|$ are shown in the left column and the phase is shown in the right column. Gray scale as in Fig. 1.

In the final frozen state, defects are confined in the center of the domain forming a rigid static chain. Shock lines appear where waves from different sides of the contour collide. The strongest shocks are attached perpendicularly to the walls and the general shock configuration is what one would expect for small symmetry breaking of the square geometry [28]. The number of defects depends on the initial condition. Solutions with no defects are also found [e.g., Figs. 2(c,d)], and are called targetlike solutions. This kind of solutions is not seen in simulations with periodic boundary conditions.

In our simulations in the square geometry with Dirichlet boundary conditions, the direction of the phase velocity (from the walls or towards the walls) and the wave number depend on the parameter values in a way that differs from the usual spiral waves in infinite systems (see Ref. [29] and Sec. III). Thus, boundaries are playing an important role in the selection of the wave speed and wave number.

In a circular domain (Fig. 3), the frozen structures are either targets (no defects) or a single central defect. Groups of defects of the same charge can also form bound states, but instead of freezing, they rotate together. This contrasts with the behavior of the square domains and is correlated with the absence of shock lines linking the boundaries to the center in the case of the circular domains. These links are probably responsible for providing rigidity to the stationary configuration in the square case. Tiny shock lines associated to small departures from circularity in the lines of constant phase can be observed also in the circle but these lines end in the bulk of the region before reaching the boundaries. On the other hand, the constant-phase lines reach the boundaries nearly tangentially in contrast to what we observe in the square. For circular domains, the phase-velocity direction changes with parameters in a way more similar to the infinite-system spiral. This is another feature revealing that circular boundaries introduce less rigidity into the pattern than square ones. The absence of corners is probably the main qualitative difference. In fact, when corners are present, they are observed to

(a)

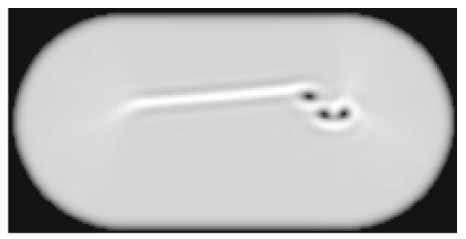

(b)

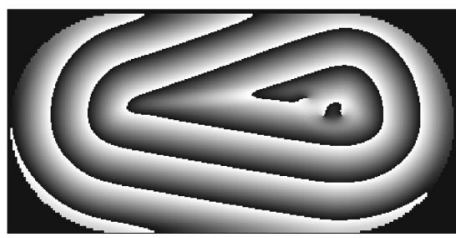

FIG. 4. Frozen structures under null Dirichlet boundary conditions in domain with stadium shape of axis $200 \times 100$ for parameter values $\alpha=2, \beta=-0.2$. Snapshots of the modulus $|A|$ are shown in (a) and the phase is shown in (b). Gray scale as in Fig. 1.

act as pacemakers from which wave emission entrains the whole system [24].

The stadium shape (Fig. 4) mixes features of the two geometries previously studied: it has both straight and circular borders. In this case, the curves of constant phase arrange themselves to combine the two behaviors described above. On the one hand the lines meet the straight portions of the border of the stadium with some characteristic angle, as it happens in square domains. However, these lines bend to become nearly tangent to the semicircles in the places where they meet with these portions of the boundaries. A typical frozen solution displays a shock line connecting the centers of the circular portions of the domain. This shock line usually contains defects and their dynamics in this stage is much slower than the annihilation that occurs in the bulk of a domain without the presence of shocks. It is also possible to find defect-free target solutions as in the case of the circle, and the behavior of the phase velocity is also similar in the way its direction can be changed by modifying the parameters.

To summarize, Dirichlet boundary conditions play a double role. On one hand, the walls naturally behave as sources (or sinks) of defects. On the other hand, a wall with null-Dirichlet conditions shows a tendency to emit plane waves that will coherently fill the whole system. The interplay between these two properties of the boundaries gives rise to interesting behavior. In the case of frozen states, the character of the walls as wave emitters dominates. The intersection of two walls (a corner) is observed also to emit waves, and the whole system becomes synchronized to this corner emission. In circular domains, on the other hand, there are no corners and wave selection is definitively dominated by the internal spirals. Another aspect of the dynamical dominance of the walls in the square case is that defects form a chain that is anchored to the boundaries by a set of shock lines; in a circle, on the contrary, the asymptotic state is usually a bound state disconnected from the boundaries.

Gaining some understanding of aspects of our numerical observations is the goal of the next sections. 


\section{SOLUTIONS IN UNBOUNDED DOMAINS}

In this section, we review some of the analytical solutions of the CGLE in unbounded domains. First we start with plane waves, continue with one-dimensional holes, and finish with two-dimensional spirals.

The CGLE possesses, among many other solutions, a family of plane-wave solutions and solutions containing phase-singular points. The plane-wave continuous family is parametrized by the corresponding wave-number $\mathbf{k}$. The form of the solutions is $A=R \exp [i(\mathbf{k} \cdot \mathbf{x}-\omega t)]$, where $R$ $=\sqrt{1-k^{2}}, \omega(k)=\beta-k^{2}(\beta-\alpha)$, and $k=|\mathbf{k}|$. The limit of stability of plane waves is known as the Benjamin-Feir line and is given by the curve $1+\alpha \beta=0$; if this quantity is positive, some stable plane wave exists [30]; if $1+\alpha \beta<0$, all plane waves are unstable. The limit is given by the stability of the plane wave with $k=0$. Stability analysis gives that plane waves possessing wave-number $k$ in the range $\left[-k_{c}\right.$, $k_{c}$ ], where $k_{c}=\sqrt{(1+\alpha \beta) /\left(3+\alpha \beta+2 \beta^{2}\right)}$, are stable. The instability is with respect to long-wavelength disturbances whose wave vectors are parallel to $\mathbf{k}$ (Eckhaus instability) [30]. It will be useful for the future discussion to have an expression for the phase velocity of the waves, and of the group velocity of small perturbations on such waves, $\mathbf{v}_{p h}$ and $\mathbf{v}_{g r}$, respectively

$$
\begin{gathered}
\mathbf{v}_{p h}=\frac{\omega(k)}{k} \hat{\mathbf{k}}, \\
\mathbf{v}_{g r}=-2 k(\beta-\alpha) \hat{\mathbf{k}} .
\end{gathered}
$$

$\hat{\mathbf{k}}$ is the unit vector in the direction of $\mathbf{k}$. The expression for the group velocity [31] turns out to be equivalent to the linearly looking expression $\mathbf{v}_{g r}=\nabla_{\mathbf{k}} \omega(k)$, even though $\omega(k)$ is the dispersion relation of nonlinear waves.

In addition to simple waves, the one-dimensional CGLE possess a one-parameter family of solutions for which the amplitude displays a region of local depression. Their analytic form was determined by Nozaki and Bekki [32], and they are, therefore, also referred to as Nozaki-Bekki solutions or holes. One member the family is characterized by the value of $A$ being zero at a point, called the core of the hole, and asymptotically behaving, at both sides of the core, as a plane wave of wave-number $k$. It is worth noting that this one-dimensional hole solution was also obtained by Hagan [29] as a subproduct of his calculations for twodimensional spirals. At variance with the other members of the Nozaki-Bekki family, this hole does not travel into the system, and thus, it will be denoted as the standing hole. Its analytical expression (choosing the origin of coordinates at the hole core) can be written as

$$
W_{H}(x, t)=\sqrt{1-k^{2}} \tanh (p x) \exp [i\{\psi(x)-\omega t\}],
$$

where $\psi$ is a function of $x$ satisfying

$$
d \psi / d x=k \tanh (p x)
$$

[i.e., $\psi=\psi_{0}+(k / p) \log \cosh (p x)$, with $\psi_{0}$ an arbitrary reference phase] and $\omega, k$, and $p$ are related according to

$$
\begin{gathered}
\omega=\beta-k^{2}(\beta-\alpha), \\
k=\frac{2 p^{2}-1}{3 p \alpha}, \\
\left\{4(\beta-\alpha)+18 \alpha\left(1+\alpha^{2}\right)\right\} p^{4}-\{4(\beta-\alpha)+9 \alpha(1+\alpha \beta)\} p^{2} \\
+\beta-\alpha=0 .
\end{gathered}
$$

If $\alpha=0$, we get

$$
\begin{gathered}
\omega=\beta\left(1-k^{2}\right), \\
p=1 / \sqrt{2}, \\
\beta=-\frac{3 k}{\sqrt{2}\left(1-k^{2}\right)} .
\end{gathered}
$$

Thus $\beta$ and $k$ have opposite sign $(\beta k<0)$, when $\alpha=0$. For any value of $\alpha$ and $\beta$, the existence of a defectlike solution fixes the value of the asymptotic wave-number $k$ and accordingly that of $\omega$. We mention here that for configurations of the frozen type, the solutions with $\alpha$ arbitrary can be obtained from the ones with $\alpha=0$ by means of a change of variables. This fact, which frequently simplifies analysis, is detailed in the Appendix.

The phase and group velocity far from the core for the one-dimensional standing hole with $\alpha=0$ are

$$
\begin{gathered}
v_{p h}=\frac{\beta\left(1-k^{2}\right)}{k}=-\frac{3}{\sqrt{2}}<0, \\
v_{g r}=-2 k \beta>0 .
\end{gathered}
$$

Thus, the propagation of the phase is towards the core of the defect independently of the value of $\beta$. However, the group velocity is directed outwards from the core of the defect. Thus, small perturbations to this solution are expelled away from the core. The case of arbitrary $\alpha$ can also be solved numerically from Eqs. (7)-(9). Given the parameters $(\alpha, \beta)$, the line where the phase velocity is zero can be found and it is plotted in Fig. 5. The group velocity turns out to be always positive (i.e., outwards from the core) for the standing hole solutions.

The two-dimensional spiral wave solutions of the CGLE are solutions winding around a defect core (i.e., a phase singularity). In polar coordinates $(r, \theta)$ around the core, they have the analytical form [29]:

$$
D(r, \theta, t)=R(r) \exp [i(\theta+S(r)-\omega t)] .
$$

This solution represents a phase pattern rotating steadily around $r=0$ with frequency $\omega$ (and frozen modulus). The amplitude $R$ is a monotonically increasing function of $r$, proportional to $r$ near the origin, and asymptotically approaching some value $R_{\infty}<1$ for large $r$. The function $S$ behaves smoothly in the neighborhood of the origin, taking the form $S \sim S_{0}+S_{1} r^{2}$. Far from the origin $S$ becomes proportional to $r$, behaving as $S \sim k r$. In this way, in the distant region, the 


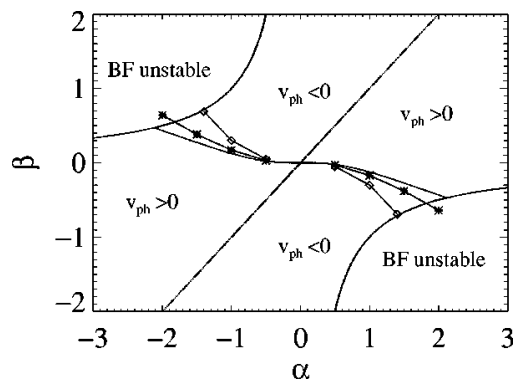

FIG. 5. Parameter space of the CGLE. Different regions are separated by solid lines: BF unstable regime where there are no stable plane-wave solutions in the infinite system; regions where the phase velocity $v_{p h}$ is positive or negative are also shown, and separated by an additional solid line for the case in which a single Dirichlet wall is present in the system (this line applies also to the one-dimensional standing hole). Star line corresponds to zero phase velocity for emission from a corner between two Dirichlet walls spanning an angle of $135^{\circ}$; diamond line corresponds to a $90^{\circ}$ corner.

isophase lines approach the form of Archimedian spirals, converging to plane waves with wave-number $k$. Thus, $R_{\infty}$ $=\sqrt{1-k^{2}}$ and $\omega=\beta-k^{2}(\beta-\alpha)$. The charge of solutions of the form Eq. (15) is, according to Eq. (2), equal to +1 . There exists also a negatively charged spiral, with the form of Eq. (15) but with $\theta$ replaced by $-\theta$. In spiral waves, wave motion is induced in such a manner as to cause the global synchronization of the medium by the defect.

It is important to notice that, in both one (the standing hole) and two dimensions (the spiral solution), imposing the requirements of zero field at the core, and plane-wave behavior far from the core, the value of $k$ gets fixed. Thus, fixing the parameter values $(\alpha, \beta)$, the spiral structure (and the standing hole) is unique (except for an arbitrary change in the location of the core). The precise way in which wavenumber, frequency, phase or group velocities depend on parameter values $(\alpha, \beta)$ can be found, for example, in Ref. [29].

\section{SOLUTIONS WITH A SINGLE WALL}

As a first step to understanding the solutions of the CGLE in bounded domains, we study in this section solutions in the presence of a single wall where the value of the complex field $A$ is set to zero. We observe numerically that, starting from random initial conditions in a bounded domain with a single Dirichlet wall, frozen solutions are reached asymptotically (see Fig. 6). In our numerical implementation, the Dirichlet wall $(A=0)$ is the left one, Neumann boundary conditions (zero normal derivative of $A$ ) are applied to the right wall, and the upper and lower limits of the domain are identified via periodic boundary conditions. We use the Neumann wall because previous numerical results [24] reveal its rather passive sink role, being able to absorb waves and defects without altering the selection mechanisms imposed by the Dirichlet wall. Initially, some (spiral) defects are formed. Typically, the Dirichlet wall starts to emit plane waves that push the defects towards the opposite boundary until they are (a)

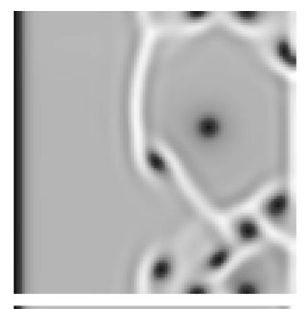

(c)

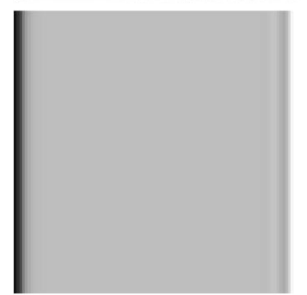

(e)

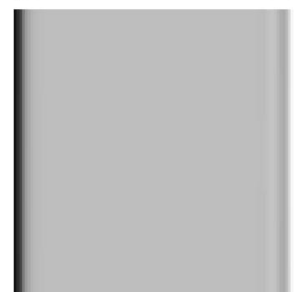

(b)

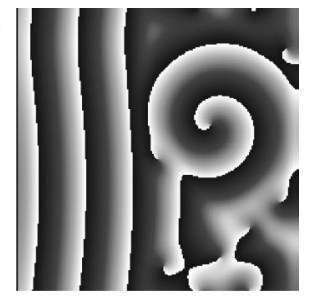

(d)

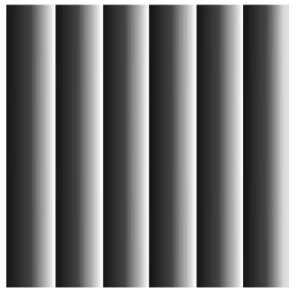

(f)

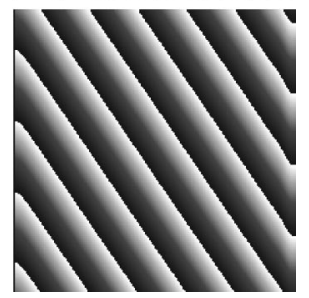

FIG. 6. Modulus (left column) and phase (right column) of the solution of Eq. (1) for $\alpha=2$ and $\beta=-0.2$ with Dirichlet boundary conditions for the left boundary, Neumann for the right one, and periodic in the horizontal ones. $(a, b)$ : Early-time state starting from random initial conditions of small amplitude.(c,d): The final asymptotic state. The lines of constant phase travel to the right. Notice that although there is a developed spiral defect, it disappears through the Neumann boundary at long times. $(e, f)$ : The long-time asymptotic state from an initial condition consisting of distorted plane wave with wave vector oblique to the boundaries. A solution of the form Eq. (16), with wave vector close to the initial one, is finally reached.

all expelled or annihilated. The stationary solution is the twodimensional extension of the one-dimensional standing hole solution described in Sec. III [that is a continuous line of holes with their cores on the wall: $\left.W_{H}(x, y, t)=W_{H}(x, t)\right]$.

We can investigate the possibility of more complex solutions in which the amplitude is independent of the $y$ direction and takes the form of a hole solution in one dimension, but with a phase that depends explicitly on both coordinates. We study first the case of $\alpha=0$ to come back later to the general case.

We look for solutions of the form

$$
W_{W}(x, y, t)=\sqrt{1-k^{2}} \tanh (p x) \exp [i\{\psi(x, y)-\omega t\}]
$$

with $\omega=\beta\left(1-k^{2}\right)$ and $k^{2}=k_{x}^{2}+k_{y}^{2}$. Assuming the form $\psi(x, y)=\psi(x)+\psi(y)$, we get the relations

$$
\begin{gathered}
\partial_{x} \psi(x, y)=k_{x} \tanh (p x), \\
\partial_{y} \psi(x, y)=k_{y}, \\
2 p^{2}=1-k_{y}^{2},
\end{gathered}
$$




$$
3 k_{x} p=\beta\left(1-k^{2}\right),
$$

and substitution of Eq. (19) into Eq. (20) gives

$$
\frac{3 k_{x} \sqrt{1-k_{y}^{2}}}{\sqrt{2}}=-\beta\left(1-k^{2}\right) \text {. }
$$

Note that if $k_{y}=0$ we recover the expression for the onedimensional standing hole solution [in particular we recover Eq. (12)].

We can perform a similar calculation for the general case of parameters $\alpha$ and $\beta$. For a solution of the form Eq. (16), Eqs. (17) and (18) remain valid, and $\omega, k$, and $p$ are related according to

$$
\begin{gathered}
\omega=\beta-k^{2}(\beta-\alpha), \\
k_{x}=-\frac{2 p^{2}+k_{y}^{2}-1}{3 p \alpha}, \\
0=-3 p k_{x}+\alpha\left(2 p^{2}-k_{x}^{2}\right)-\beta\left(1-k^{2}\right) .
\end{gathered}
$$

In contrast with the selection mechanism for the standing hole or the spiral solutions, the presence of the wall does not select a unique wave vector, but a one-parameter family of solutions parametrized by either $k_{x}$ or $k_{y}$ arises instead from Eqs. (17)-(21) or (22)-(24) for given values of $\alpha$ and $\beta$. Different solutions in the family differ in the direction and magnitude of the wave-vector k. Different wave vectors change the angle of intersection between the lines of constant phase and the wall. Figures $6(\mathrm{e}-\mathrm{f})$ are the final state in a numerical simulation in which the initial condition was close to Eq. (16) with $\mathbf{k}$ oblique with respect the wall. The displayed state is identical (far enough from the Neumann wall) to Eq. (16) with Eqs. (22)-(24) thus numerically proving the stability of this solution. Different orientations of $\mathbf{k}$ can be tested in the same way. However, if starting with random initial conditions, we typically find solutions corresponding to the case $k_{y}=0$ [Figs. $\left.6(\mathrm{c}-\mathrm{d})\right]$ that is the simplest twodimensional extension $\left(k_{y}=0\right)$ of the standing hole.

The prevalence of the $k_{y}=0$ solution when starting from random initial conditions (of small amplitude) can be understood from the characteristics of the linear instability of the state $A=0$, and from the effect of the periodic boundary conditions used in the walls perpendicular to the Dirichlet one in our simulations (horizontal walls in Fig. 6): The periodic conditions imply that the $y$-winding number $n_{y}$, defined by an integral similar to Eq. (2) in which the path $\Gamma$ stars and ends in corresponding points of the horizontal boundaries, is quantized, and it remains constant if defects are not created. This is the case in the parameter range considered here after a short transient of linear evolution in which the nonlinear state is developed. In consequence, the $n_{y}$ of the final state is determined by the value of this quantity at the end of the linear regime. No such rigidity occurs along $x$ because of the nonperiodic conditions used. The linear regime in which the pattern grows starting from $A \approx 0$ can be analyzed by neglecting the nonlinear term in Eq. (1) and introducing the ansatz $A(x, y, t)=f(x, y) e^{\epsilon t}$. By separation of variables, and imposing periodicity in the direction $y$, finiteness at large $x$, and the Dirichlet condition at $x=0$, one finds that the linear eigenmodes are of the form $f_{k_{x}, k_{y}}(x, y)=e^{k_{y} y} \sin \left(k_{x} x\right)$, with eigenvalues $\epsilon=1-(1+i \alpha)\left(k_{x}^{2}+k_{y}^{2}\right)$. Thus, eigenmodes with small wave numbers grow faster and will dominate at the beginning of the nonlinear saturation regime, as is indeed observed. The periodic boundary conditions in the $y$ direction quantize the value of $k_{y}$ of the eigenmodes, thus forbidding any small $k_{y}$ different from zero. $k_{y}=0$ gives $n_{y}=0$, and this value will be preserved by the nonlinear regime. In consequence, typically $k_{x}$ will adapt to the nonlinearly imposed value given by Eqs. (22)-(24) for constant $k_{y}=0$. These arguments are confirmed by numerical simulations with three Neumann walls and one Dirichlet one, in which tilted waves with small, but not always zero, values of $k_{y}$ are obtained outside the boundary layers around de Neumann walls.

We note that, although solutions (16) represent emission at an angle with the wall, the analytic expression predicts a small boundary layer (of size $p^{-1}$ ) in which the wave number leaves its asymptotic orientation to become parallel to the wall, so that isophase lines arrive perpendicular to the boundary. This is observed in the numerical solutions (see, for example, Fig. 6(f), and also the rectilinear walls of Figs. 1,2 , and 4) thus, nicely confirming the relevance of the analytical solutions of the observed configurations. In the case of wave emission perpendicular to the wall (and thus, wave number also perpendicular to it), the analytical expressions imply that this wave number also vanishes at the boundary. Thus, as a rule, the wave-vector component perpendicular to the wall always vanishes at the wall. We do not observe an analogous vanishing of the wave-number component perpendicular to the wall in the case of curved walls. In the square, however, conflict between the orientation emitted by neighboring walls occurs, and the exact expression (16) is appropriate only near each wall. The conflict between neighboring walls is resolved at long times by emission from the corner, as will be seen in the next section.

Another important kind of solutions with a single wall is the one that appears with Neumann boundary conditions. The solutions observed close to the right wall in Figs. 6(c-f) are of this type. These solutions have been already analyzed in the literature, especially in the context of interactions between spirals. The reason is that a Neumann wall acts as a reflecting boundary, so that having a wave impinging into the boundary is equivalent to the interaction between two sources of waves located symmetrically with respect to the wall [6]. Despite the interest of such solutions, no exact analytical expression has been found for them. Analytical solutions have been obtained, however [33], by solving the phase equation that approximates the phase dynamics for small amplitude perturbations (see Sec. V B). In agreement with the numerical observations, the solution presents a maximum modulus at the wall (a shock) and the isophase lines, straight in the far field, deform when entering a boundary layer close to the wall to arrive perpendicular (for tilted far-field incidence) to the wall. We will see in the following that these "tilted Neumann waves" are of relevance when corners are present. 


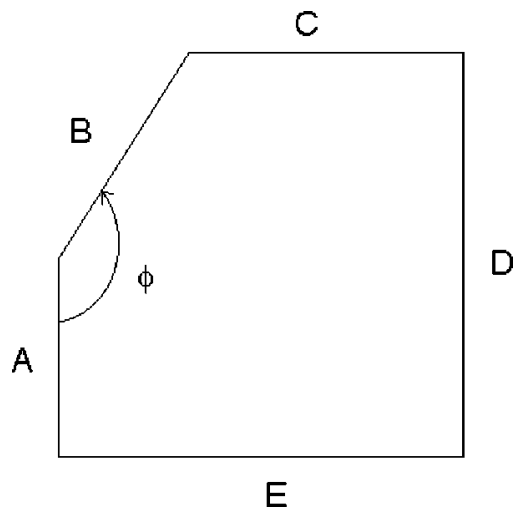

FIG. 7. Domain and boundary conditions for the simulations in Sec. V. In $A$ and $B$, null-Dirichlet boundary conditions; $C, D$, and $E$, null-Neumann boundary conditions.

It is clear on physical grounds, and confirmed by the analytical expressions from the phase approximation, that the Neumann wall can act as a sink of waves of arbitrary farfield orientation and wavelength (the maximum modulus at the shock will adapt accordingly). Neumann waves constitute, then, a biparametric family for fixed $(\alpha, \beta)$, and Neumann walls do not impose special selection mechanisms.

\section{SOLUTIONS IN PRESENCE OF CORNERS}

We now pay attention to the effects induced by the presence of corners, i.e., how the solutions adapt to the emission of two semi-infinite lines. In Sec. V A we will show that the phase velocity not only depends on the parameters of the CGLE $\alpha$ and $\beta$, but also on the angle $\phi$ between the walls of the boundary. In Sec. V B, we will present solutions of the phase equation representing wave collision; they are usually called $V$ solutions. These solutions should be matched with the boundaries, which provide selection mechanisms for the wave pattern.

\section{A. Phase velocity dependence on the angle at the boundary}

We have performed numerical simulations of the CGLE in the domain depicted in Fig. 7, where one of the walls is a broken line with a corner of a definite angle $\phi$. The boundary conditions are the following: for the right, upper, and bottom walls, Neumann boundary conditions (null-normal derivative). For the left boundary (where the corner is present), null-Dirichlet conditions. This left boundary is a line that is broken forming a variable angle $\phi$. If this angle is $180^{\circ}$, there exist the two-dimensional extensions of the standing hole described in the previous section. As the angle decreases, the wave is not longer plane, and the phase velocity adapts to the new geometry. The wave fronts may become just slightly distorted from straight lines (as in Fig. 8) or display a kink (similar to the situation in Fig. 2) depending on $\alpha, \beta$, and $\phi$. In any case, the kink is never too strong and departures from straight wave fronts never large. Changing parameters the phase velocity may vanish. The locus in parameter space where this happens is a $2 \mathrm{D}$ surface in the $(\alpha, \beta, \phi)$ space. Projections in the $(\alpha, \beta)$ plane for $\phi$ (a)

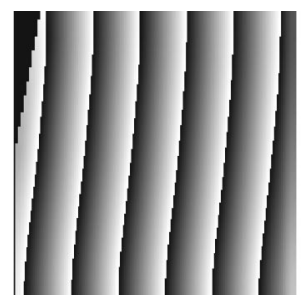

(b)

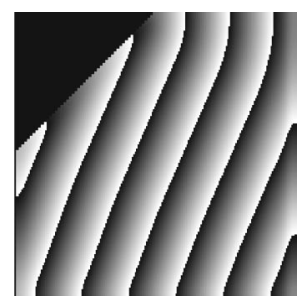

FIG. 8. Phase of the solution of Eq. (1) in gray scale, for parameter values $\alpha=2, \beta=-0.2$. In (a) the angle $\phi=\pi / 2$ $+\tan ^{-1}(1 / 5)$; (b) $\phi=3 \pi / 4$.

$=180^{\circ}$ [obtained from Eq. (5) $]$ and $\phi=135^{\circ}$ (from numerical simulation) are plotted in Fig. 5. $\phi=90^{\circ}$ corresponds to a square and is also plotted in Fig. 5. We do not see differences between squares with two or four Dirichlet walls.

Summarizing, for frozen structures, the presence of Dirichlet walls establish a selection mechanism different from the associated to the presence of a spiral core in an infinite domain (Sec. III). When the Dirichlet wall is broken, it is seen in the earliest stages of wave-pattern development that emission with isophases nearly parallel to the walls is initiated, but collision between the waves from different walls arises and a distinct final state, with wave number, phase velocity, etc., fixed by $(\alpha, \beta, \phi)$ is reached. We now investigate how this may happen.

\section{B. $V$ solutions of the phase equation and pattern selection}

For unbounded domains and for small amplitude modulations, a phase description of the complex field $A$ can be made. The approximate equation for the phase is [15]

$$
\dot{\varphi}=\omega_{0}+b_{0} \nabla^{2} \varphi+b_{1}(\nabla \varphi)^{2}
$$

where $b_{0}=1+\alpha \beta, b_{1}=(\beta-\alpha)$, and $\omega_{0}=-\beta$. The modulus $R$ of the solution is slaved to the phase as $R^{2} \approx 1$ $-(\nabla \varphi)^{2}-\alpha \nabla^{2} \varphi$.

We look for solutions $\varphi=\varphi(x, y, t)$ representing phase waves with nonstraight isophase lines. This is what is observed when Dirichlet waves from different walls interact (Figs. 2,8). Analytic expressions of this type are known for the phase equation: the $V$ solutions [15]. Since we are for the moment considering an unbounded system, the position of the shock is arbitrary. But in order to use a notation useful for the next cases, we assume that the shock is at the diagonal $x=y$ of an arbitrary cartesian frame. We impose different but symmetric wave vectors far from the shock, that is $\nabla \varphi$ $\rightarrow\left(k_{1}, k_{2}\right)$ if $x \ll y$, and $\nabla \varphi \rightarrow\left(k_{2}, k_{1}\right)$ if $y \gg x$, thus, getting the family of solutions:

$$
\begin{aligned}
\varphi(x, y, t)=[ & \left.\omega_{0}+b_{1}\left(k_{1}^{2}+k_{2}^{2}\right)\right] t+\frac{k_{1}+k_{2}}{2}(x+y) \\
& +\frac{b_{0}}{b_{1}} \log \left[\cosh \left(\frac{b_{1}}{b_{0}} \frac{k_{1}-k_{2}}{2}(x-y)\right)\right] .
\end{aligned}
$$

The spatial dependence of this solution can be separated in terms of the variables $u=x+y$ and $v=x-y$, and thus, the phase equation is also separable in $u$ and $v$. The change from 
$(x, y) \rightarrow(u, v)$ is a rotation bringing the shock line to one of the axes. After inspection of the derivatives normal to the shock, we see that half of these $V$ solutions can be interpreted as tilted waves approaching a Neumann wall at the shock position, being the other half just a specular image. As for Neumann tilted waves, we have a biparametric family, parametrized by $k_{1}$ and $k_{2}$.

As solutions of the phase equation, the $V$ solutions are strictly valid only far from the boundaries, where the modulus of the field remains nearly constant. Matching to solutions of the form of Eq. (16) should be performed close to Dirichlet boundaries. We know (Sec. IV) that for this type of boundaries, the two components of the far-field wave vector are not independent [Eqs. (22)-(24)]. For definiteness, let us consider two Dirichlet walls along the axes $x$ and $y$, thus meeting at a $90^{\circ}$ corner. Matching to one of the walls introduces a relationship between $k_{1}$ and $k_{2}$ in Eqs. (26). The shock line $x=y$ bisects the angle between the walls, and by symmetry, no additional constraints appear from matching to the other boundary. Thus, one of the parameters in the $V$ solution, which can be taken as the angle between the two waves, is still undetermined. From the numerical simulations, it appears that this angle becomes determined when the medium is synchronized by the waves coming from the corner between the two walls. We do not have a rigorous argument to demonstrate that this is the case, but the following heuristic argument is a step towards such a demonstration: Close to the walls, a phase description is no longer valid, and the modulus approaches zero. The solution is of the frozen type, which we write as $A(x, y, t)=R(x, y) \exp [i(\psi(x, y)$ $-\omega t)]$ with real $R, \psi$, and $\omega$. Since this solution should become Eq. (26) far from the walls, we immediately find

$$
\omega=\omega_{0}+b_{1}\left(k_{1}^{2}+k_{2}^{2}\right)=-\beta+(\beta-\alpha)\left(k_{1}^{2}+k_{2}^{2}\right) .
$$

Sufficiently close to the corner at $x=y=0$, the modulus $R$ is small. Writing for it and for $\psi$ a Taylor expansion, imposing symmetry across the $x=y$ line, and substituting into the CGLE (1), we easily find at the lowest order in distance to the corner the following behavior (we only write down the expressions for $\alpha=0$ ):

$$
\begin{gathered}
R(x, y) \approx B x y, \\
\psi(x, y) \approx \psi_{0}-\frac{\omega}{12}\left(x^{2}+y^{2}\right) .
\end{gathered}
$$

Close to the walls, the local wave vector is $\mathbf{q}=\nabla \psi=$ $-(\omega / 6)(x, y)$, so that in the diagonal $\mathbf{q}_{d}=-(\omega / 6)(x, x)$ with modulus $q_{d}=(x \omega) / \sqrt{1} 8$. Far from the corner, this wave number should match the one obtained from the $V$-solution $\mathbf{k}_{d}=\left(k_{1}+k_{2}\right) / \sqrt{2}$. An approximate way of doing this is imposing that both wave numbers become equal at some distance $x \approx a$ from the corner:

$$
\frac{\omega}{\sqrt{18}} a \approx \frac{k_{1}+k_{2}}{\sqrt{2}}
$$

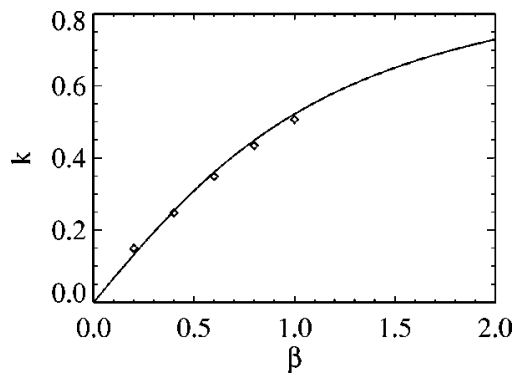

FIG. 9. Modulus of $\mathbf{k}$ versus parameter $\beta$ (for a square with Dirichlet walls, and $\alpha=0$ ) obtained from our theoretical arguments [Eqs. (22)-(24) and $k_{x}=k_{y}$, solid line] and direct numerical simulation (diamonds). Around $\beta \simeq 1.15$, the frozen state becomes unstable.

$a$ is an unknown constant of the order of the boundary layer size $\left(p^{-1}\right)$. For given parameter values $\alpha$ and $\beta$ this expression gives an extra relationship between $k_{1}$ and $k_{2}$ or, equivalently, between the modulus $k$ and the angle of emission from the walls. This, and Eqs. (22-24), completely fixes the solution in the presence of a corner.

Of course, precise numerical values cannot be obtained since $a$ is unknown, but the previous heuristic argument was intended only to illustrate how the presence of the corner resolves the conflict between the neighboring waves, and fixes the wave pattern as numerically observed. For situations such as the ones depicted in Fig. 8 for which the wave fronts remain relatively straight, we have $k_{1} \approx k_{2}$, which can be used as a substitute of Eq. (30) to fix the pattern. In fact, this is never a bad approximation. For example, from a $90^{\circ}$ corner, straight and symmetric wave fronts indicates wave emission at $45^{\circ}$ from each wall. We have checked that this is in fact equivalent to Eq. (30) with $a^{2}=8$. Since this value of $a$ is within the boundary layer range $\left(p^{-1}=\sqrt{2}\right.$ for $\left.\alpha=0\right)$, both approaches [Eq. (30) and $k_{1} \approx k_{2}$ ] are mutually consistent and they can be thought as two different approximations to the same fact that the corner fixes the wave number. Assuming $k_{1}=k_{2}$, we have plotted in Fig. 9 a comparison between the results from the numerical simulations and the analytical prediction $k^{2}=1-\left(\sqrt{1+8 / 9 \beta^{2}}\right)^{-1}$ for $\alpha=0$. The agreement is good and confirms the relevance of the walls and corners into the wave selection process, and justifies the ansatz made in the derivation of the theoretical results.

The above arguments have been developed from expressions (28)-(29), which require $\alpha=0$. The property explained in the Appendix implies that the selection mechanism identified here will also be present at any value of $\alpha$. Equations (28)-(29) are also restricted to a $90^{\circ}$ corner. The methodology may be applied to other angles by replacing these expressions by the corresponding behavior at short distances from the corner. For example, if $\alpha=0$, smallness of $R$ and of the wave number (at Dirichlet walls, the wave-vector component perpendicular to the wall vanishes, so that both components will vanish at a corner) reduces the equation for the modulus $R$ to $\nabla^{2} R+R \approx 0$. This equation is easily solved by separation of variables in polar coordinates $(r, \theta)$ centered at the corner of angle $\phi$ to give 


$$
R \approx r \pi \phi \sin \left(\frac{\pi}{\phi} \theta\right),
$$

which is the substitute of Eq. (28) for arbitrary angle.

\section{CONCLUSIONS}

In this paper we have presented numerical results on the influence of boundaries in the wave-pattern selection of a self-oscillatory medium, in parameter regimes in which frozen structures are reached. Analytical solutions in the presence of walls and corners have been presented and shown to be relevant to the numerically observed configurations. The dominance of Dirichlet walls, the relative passive role of Neumann walls, and the synchronization properties of corners, are possibly generic features that should be found in other self-oscillatory model systems. Extrapolation to real experimental oscillatory media should be made with care, however, since determining the correct boundary conditions applying to the amplitude equation associated to a particular medium is a subtle task [25,26].

Note added. In the following address we have made available a web page containing simulations of the CGLE in different geometries related to this paper: http:// www.imedea.uib.es/Nonlinear/research_topics/cglwalls

\section{ACKNOWLEDGMENTS}

Financial support from DGES, Spain, Project Nos. CONOCE BFM2000-1108 and PB97-0141-C01-01, is greatly acknowledged. V.M.E. acknowledges financial support from the Danish Natural Science Research Council.

\section{APPENDIX}

The equation resulting from restricting the CGLE Eq. (1) to frozen solutions of the form $A(\mathbf{x}, t)=f(\mathbf{x}) e^{-i \omega t}$, with $\omega$ a real frequency and $f$ a possibly complex function of the position, admits a change of variables [29] that transforms the case with parameters $(\alpha, \beta)$ into the case with parameters $\left(0, \beta^{\prime}\right)$. The transformation is

$$
\begin{gathered}
\beta^{\prime}=\frac{\beta-\alpha}{1+\alpha \beta}, \\
\omega^{\prime}=\frac{\omega-\alpha}{1+\omega \alpha}, \\
\mathbf{x}^{\prime}=\mathbf{x} \sqrt{\frac{1+\alpha \omega}{1+\alpha^{2}}}=\frac{\mathbf{x}}{\sqrt{1-\alpha \omega^{\prime}}}, \\
f^{\prime}=f \sqrt{\frac{1+\alpha \beta}{1+\omega \alpha}} .
\end{gathered}
$$

Obtaining a frozen solution (i.e., a function $f^{\prime}\left(\mathbf{x}^{\prime}\right)$ and an associated frequency $\omega^{\prime}$ ) at parameters $\alpha=0$ and $\beta^{\prime}$, thus, allows finding corresponding solutions $(f, \omega)$ at arbitrary values of $\alpha$ and the corresponding $\beta=\left(\beta^{\prime}+\alpha\right) /\left(1-\alpha \beta^{\prime}\right)$. This useful relationship has been used along this paper to generate solutions at arbitrary parameters from easier solutions at $\alpha=0$. Note that if $f^{\prime}$ contains a factor of the form $e^{i \mathbf{k}^{\prime} \cdot \mathbf{x}^{\prime}}$, then $f$ will have a factor of the form $e^{i \mathbf{k} \cdot \mathbf{x}}$, with $\mathbf{k}$ $=\mathbf{k}^{\prime} / \sqrt{1-\alpha \omega^{\prime}}$.
[1] M. C. Cross and P. C. Hohenberg, Rev. Mod. Phys. 65, 851 (1993).

[2] M. C. Cross and P. C. Hohenberg, Science 263, 1569 (1994).

[3] M. Dennin, G. Ahlers, and D. S. Cannell, Science 272, 388 (1996)

[4] D. A. Egolf, I. V. Melnikov, W. Pesch, and R. E. Ecke, Nature (London) 404, 733 (2000).

[5] W. van Saarloos, in Spatiotemporal Patterns in Nonequilibrium Complex Systems, edited by P. E. Cladis, P. PalffyMuhoray (Addison-Wesley, Reading, 1994).

[6] I. S. Aranson, L. Kramer, and A. Weber, Phys. Rev. E 47, 3231 (1993); ibid. 48, R9 (1993), and references therein.

[7] P. Kolodner, S. Slimani, N. Aubry, and R. Lima, Physica D 85, 165 (1995).

[8] P. Coullet, L. Gil, and F. Roca, Opt. Commun. 73, 403 (1989).

[9] M. San Miguel, Phys. Rev. Lett. 75, 425 (1995).

[10] Y. Kuramoto and T. Tsuzuki, Prog. Theor. Phys. 52, 356 (1974)

[11] Y. Kuramoto and S. Koga, Prog. Theor. Phys. Suppl. 66, 1081 (1981).

[12] T. Leweke and M. Provansal, Phys. Rev. Lett. 72, 3174 (1994).

[13] R. Schielen, A. Doelman, and H. de Swart, J. Fluid Mech. 252, 325 (1993)
[14] P. C. F. van der Vaart and H. K. Dijkstra, Phys. Fluids 9, 615 (1993).

[15] Y. Kuramoto, in Chemical Oscillations, Waves, and Turbulence (Springer-Verlag, Berlin, 1984).

[16] A. T. Winfree, in When Time Breaks Down (Princeton University Press, Princeton, 1987).

[17] I. Rehberg, S. Rasenat, and V. Steinberg, Phys. Rev. Lett. 62, 756 (1989).

[18] K. Staliunas, G. Slekys, and C. O. Weiss, Phys. Rev. Lett. 79, 2658 (1997).

[19] I. Aranson, D. Hochheiser, and J. V. Moloney, Phys. Rev. A 55, 3173 (1997).

[20] B. J. Gluckman and C. B. Arnold, and J. P. Gollub, Phys. Rev. E 51, 1128 (1995).

[21] V. M. Eguíluz and P. Alstrøm, E. Hernández-García, and O. Piro, Phys. Rev. E 59, 2822 (1999); e-print chao-dyn/9805003.

[22] V. M. Eguíluz, E. Hernández-García, O. Piro, and S. Balle, Phys. Rev. E 60, 6571 (1999); e-print chao-dyn/9906014.

[23] V. M. Eguíluz, E. Hernández-García, and O. Piro, Physica A 283, 48 (2000); e-print nlin.CD/0004029.

[24] V. M. Eguíluz, E. Hernández-García, and O. Piro, Int. J. Bifurcation Chaos Appl. Sci. Eng. 9, 2209 (1999); e-print chao-dyn/9812010. 
[25] A. J. Roberts, J. Aust. Math. Soc. B, Appl. Math. 34, 54 (1992).

[26] C. Martel and J. M. Vega, Nonlinearity 9, 1129 (1996).

[27] The numerical code used to solve the time evolution of the complex field $A(\mathbf{x}, t)$ in Eq. (1) in two dimensions with nullDirichlet boundary conditions and for arbitrary geometries is as follows. First of all, a rectangular grid containing the desired spatial domain is defined. Secondly, external grid points are maintained fixed at a value $A=0$; internal points are subjected to the discretized version of Eq. (1) where the Laplacian is considered up to second order and the time evolution is solved using a fourth order Runge-Kutta method (typical time step $10^{-2}$ ). In particular, for the study of corners, we consider grids that contain points exactly at the corner.

[28] T. Ondarcuhu, G. B. Mindlin, H. L. Mancini, and C. Pérez García, Phys. Rev. Lett. 70, 3892 (1993); D. Maza, B. Echebarria, C. Perez-Garcia, and H. Mancini, Phys. Scr., T 67, 82 (1996).
[29] P. S. Hagan, SIAM (Soc. Ind. Appl. Math.) J. Appl. Math. 42, 762 (1982).

[30] The stability mentioned here is just with respect to perturbations of long wavelength. It is known that additional instabilities, at finite wave number, may appear even if $1+\alpha \beta>0$; see, for example, R. Montagne and P. Colet, Phys. Rev. E 56, 4017 (1997); these instabilities are not of relevance for the present work.

[31] R. Montagne, E. Hernández-García, A. Amengual, and M. San Miguel, Phys. Rev. E 56, 151 (1997).

[32] K. Nozaki and N. Bekki, J. Phys. Soc. Jpn. 53, 1581-1582 (1984); K. Nozaki and N. Bekki, Phys. Lett. A 110, 133 (1985).

[33] V. N. Biktashev, in Nonlinear Waves II, edited by A. V. Gaponov-Grekhov and M. I. Rabinovich (Springer, Heidelberg, 1989); I. S. Aranson, L. Kramer, and A. Weber, Physica D 53, 376 (1991). 